\title{
NUEVAS MEDIDAS CONTRA LOS COMBATIENTES TERRORISTAS EXTRANJEROS EN EUROPA: ¿UN MODELO PARA FILIPINAS?*
}

\author{
New measures against foreign terrorist fighters in Europe: \\ a model for the Philippines?
}

\author{
Alejandro Sánchez Frías \\ Investigador Predoctoral FPU en Derecho Internacional Público \\ Universidad de Málaga \\ asanchezfrias@uma.es
}

doi: http://dx.doi.org/10.18543/ed-66(1)-2018pp317-333

Recibido: 12.04 .2018

Aceptado: 25.06.2018

\section{Resumen}

Los combatientes terroristas extranjeros se han convertido en una dolorosa realidad para la comunidad internacional en los últimos años. El Consejo de Seguridad de

* Cómo citar / Citation 'Chicago-Deusto' (Autor-fecha / Author-date / Lista de referencias / Reference list entries): Sánchez Frías, Alejandro. 2018. «Nuevas medidas contra los combatientes terroristas extranjeros en Europa: ¿un modelo para Filipinas?». Estudios de Deusto 66, n. ${ }^{\circ}$ 1: 317-333. doi: http://dx.doi.org/10.18543/ed-66(1)-2018pp317-333.

Doctorando en el Área de Derecho Internacional Público y Relaciones Internacionales de la Universidad de Málaga, Máster en Estudios de la Unión Europea (Universidad de Salamanca) y Máster en Derecho Europeo (Colegio de Europa, Brujas). El presente artículo se enmarca en el programa de ayudas de investigación FPU del Ministerio de Educación, Cultura y Deporte (FPU-2015/06775), así como en el proyecto del Ministerio de Economía y Competitividad «Los muros en el Derecho Internacional contemporáneo: consecuencias para la seguridad, la dignidad humana y la sostenibilidad» (DER-201565486-R) y el Grupo de Investigación «Protección Internacional de los Derechos Humanos, Seguridad y Medioambiente (SEJ-593)» del Plan Andaluz de Investigación. 
Naciones Unidas ya ha solicitado a los Estados que adapten sus ordenamientos legislativos para hacerles frente. Europa ha sido una de las víctimas de este fenómeno y ya se han adoptado medidas por el Consejo de Europa y la Unión Europea en diversos ámbitos, siendo uno de los principales el de la armonización de tipos penales para criminalizar las conductas típicas de los combatientes terroristas extranjeros. Filipinas, también acosada por este problema, no ha adoptado aún medidas específicas y puede prestar atención a las iniciativas europeas. Sin olvidar, eso sí, que las injerencias que conllevan en los derechos humanos y las libertades fundamentales deben estar sujetas a controles y garantías.

\section{Palabras clave}

Combatientes; terroristas; extranjeros; Consejo de Seguridad; conductas típicas.

\section{Abstract}

Foreign terrorist fighters have become a painful reality for the internacional community in recent years. The United Nations Security Council has asked the States to adapt their legislation to combat this issue. Europe has been one of the victims of this phenomenon and the Council of Europe and the European Union have already taken action in different areas, one of the main being the harmonization of criminal codes to punish the criminal acts of these foreign terrorist fighters. The Philippines, also harassed by this problem, hasn't adopted any specific step and can pay attention to the european iniciatives. Of course, we cannot forget that the interference in human rights and fundamental freedoms needs to be subject to controls and guarantees.

\section{Keywords}

Fighters; terrorists; foreigns; The United Nations Security Council; criminal acts. 


\begin{abstract}
SUMARIO: I. INTRODUCCIÓN. II. COMBATIENTES TERRORISTAS EXTRANJEROS: UNA AMENAZA COMÚN PARA EUROPA Y FILIPINAS. III. LA RESPUESTA JURÍDICA INTERNACIONAL. 1. El recurso a las medidas penales en el Ámbito Europeo. 2. El impacto de la criminalización de ciertas conductas en las libertades fundamentales. 4. CONCLUSIONES. BIBLIOGRAFÍA.
\end{abstract}

\title{
I. INTRODUCCIÓN
}

La participación cada vez mayor de nacionales y residentes de países europeos en las filas de grupos terroristas en el extranjero, así como la comisión de atentados a su retorno, ha evidenciado la necesidad de adoptar medidas comunes en la lucha contra una amenaza que no conoce de fronteras. Siguiendo las líneas directrices de la Resolución 2178 del Consejo de Seguridad de Naciones Unidas (CSNU), tanto el Consejo de Europa como la Unión Europea (UE) han desarrollado instrumentos para combatir este fenómeno. Entre ellos se encuentra la armonización de las legislaciones penales para criminalizar las conductas relacionadas con lo que el antiguo director del FBI, James Comey, ha identificado con una «terrorist diaspora» tras la pérdida cada vez mayor de territorios por el Daesh $^{1}$. Al igual que muchos países europeos, Filipinas también ha sido víctima de los denominados «combatientes terroristas extranjeros», hasta el punto de tener una de sus ciudades asediada por grupos afines al Daesh. Pero al contrario que las organizaciones regionales europeas, Filipinas no ha tomado medidas expresas para implementar la Resolución 2178. En las siguientes páginas se examinará brevemente el fenómeno de los combatientes terroristas extranjeros en Europa y Filipinas y el contenido de los instrumentos adoptados en Europa y las críticas que reciben, lo cual permitirá valorar su utilidad en el territorio insular y los retos que plantearía desde el punto de vista de la protección de los derechos humanos y las libertades fundamentales.

\section{COMBATIENTES TERRORISTAS EXTRANJEROS: UNA AMENAZA COMÚN PARA EUROPA Y FILIPINAS}

La primera organización internacional en expresar sus preocupaciones sobre la integración de los combatientes extranjeros en organizaciones terroristas fue la Organización Internacional de Policía Criminal (INTERPOL,

${ }^{1}$ Gerstein y Scholtes 27 de septiembre de 2016. 
por contracción de la expresión inglesa)². Pero el término «combatiente terrorista extranjero» dio el salto a la fama en la esfera internacional con la conocida Resolución 2178 del CSNU, que lo define como aquella persona que «viaja a un Estado distinto de su Estado de residencia o nacionalidad con el propósito de cometer, planificar o preparar actos terroristas o participar en ellos, o de proporcionar o recibir adiestramiento con fines de terrorismo, incluso en relación con conflictos armados $»^{3}$.

Los atentados que tuvieron lugar en Francia y Bélgica durante el año 2015 hicieron que el combatiente terrorista extranjero pasase de riesgo a realidad para la sociedad europea ${ }^{4}$. Y es que a los más de quince mil combatientes extranjeros que ya se encontraban en zonas de conflicto durante el año 2016 hay que sumar los denominados «foreign fighter wannabes», es decir, aquellos que no forman parte de una organización terrorista pero se inspiran en ellas para la comisión de atentados ${ }^{5}$. En ese mismo año Europa ocupaba el tercer puesto en el envío de combatientes extranjeros a las zonas de conflicto en Siria e Irak (alrededor de 5,000 nacionales o residentes) con Francia, Reino Unido, Alemania y Bélgica a la cabeza de este aciago ranking ${ }^{6}$.

Pero la sociedad europea no es la única afectada por el fenómeno de los combatientes terroristas extranjeros. La situación de las Filipinas es también delicada en este aspecto. El fracaso en la aprobación de la Bangsamoro Islamic Law a principios de febrero de 2016 fue vista por algunos analistas como un alimento más de esa sensación que tiene la comunidad musulmana de ser víctimas de continuas injusticias y que la acercaría al terrorismo del Daesh, algo enseñado y repetido a una juventud que es testigo diario de la violencia ${ }^{7}$.

De hecho, ciertos grupos terroristas filipinos han mostrado su apoyo a la causa del Daesh. Los conocidos como Bangsamoro Islamic Freedom Fighters (BIIF) declararon su pleitesía al Daesh a través de vídeos ${ }^{8}$, así como miembros del Abu Sayyaf Group (ASG) en los siguientes términos: "We pledge bay'ah to Caliph Sheikh Abu Bakr al-Baghdadi Ibrahim Awwad AlQurashi Al-Husseini for loyalty and obedience in adversity and comfort. We

2 INTERPOL, «Los combatientes extranjeros, eje central de una reunión de INTERPOL sobre la lucha contra el terrorismo».

${ }^{3}$ Consejo de Seguridad de Naciones Unidas, Resolución 2178 de 24 de septiembre de 2014, Doc. S/RES/2178.

${ }^{4}$ LÓPEZ-JACOISTE 2016: 51.

5 Paulussen 2016: 3.

${ }^{6}$ Munich Security Conference, «Munich Security Report 2016: Boundless Crises, Reckless Spoilers, Helpless Guardians», p. 21. Disponible en: https://www.securityconference.de/fileadmin/MunichSecurityReport/MunichSecurityReport_2016.pdf (Consultado el 24 de agosto de 2017).

7 Koruth Samuel 2016: 81-100.

${ }^{8}$ Charithie Agosto de 2015: 6-7. 
pledge to obey him on anything which our hearts desire or not and to value him more than anyone else. We will not take any emir other than him unless we see in him any obvious act of disbelief that could be questioned by Allah in the hereafter»?.

A la presencia de grupos terroristas en territorio filipino y la influencia que ejercen en la población se ha sumado el miedo a que algunos de los nacionales filipinos que trabajan cerca de zonas de conflicto sean reclutados por el Daesh y enviados de vuelta a Filipinas para aplicar las técnicas aprendidas $^{10}$. En 2016 fueron accesibles al público los primeros informes alertando de la presencia de nacionales filipinos que estaban siendo entrenados por el Daesh en territorio iraquí1 ${ }^{11}$. No obstante, las declaraciones oficiales del momento pedían prudencia a la hora de conectar el terrorismo en Filipinas con el de Daesh: «the Philippines still faces the same problems: terrorist groups like the Abu Sayyaf and the Bangsamoro Islamic Freedom Fighters which are doing basically the same things, but now attributing it to their joining IS, which doesn't necessarily mean that they are IS» ${ }^{12}$.

Existían dudas en un principio sobre esta conexión entre los grupos terroristas filipinos y el Daesh. Y, junto a ello, sobre la amenaza de nacionales filipinos que retornaran a su lugar de origen tras haber sido radicalizados, así como de combatientes terroristas extranjeros que se dirigieran a este país insular para cometer sus atentados. Los acontecimientos ocurridos más recientemente obligan por desgracia a confirmar estos temores. Desde el punto de vista propagandístico, la presión a la que está sometida el Daesh en Siria e Irak ya había llevado a algunos de sus miembros a pedir que «if you cannot go to Syria, join up and go to the Philippines»y a la proclamación de una wilayah (provincia) del Daesh en abril de $2016^{13}$. Desde el punto de vista fáctico, se añade en mayo de 2017 el asedio por parte de terroristas tanto nacionales como extranjeros de la ciudad de Marawi en lo que algunos han llegado a comparar con las batallas de Aleppo, Mosul y Raqqa ${ }^{14}$.

\section{LA RESPUESTA JURÍDICA INTERNACIONAL}

En la ya citada Resolución 2178 el CSNU expresó su preocupación por «el hecho de que los combatientes terroristas extranjeros aumentan la intensidad, duración e insolubilidad de los conflictos y también pueden

\footnotetext{
9 Ressa 4 de agosto de 2014

10 Koruth Samuel, cit. Supra: 95-96.

11 Saludo 8 de octubre de 2016.

12 Esmaquel 25 de septiembre de 2014.

13 Singh y RAmaKrishna 2016.

14 Gunaratna 2017: 4.
} 
representar una seria amenaza para sus Estados de origen, los Estados por los cuales transitan y los Estados a los que viajan, así como para los Estados vecinos de las zonas de conflicto armado en las que los combatientes terroristas extranjeros realizan sus actividades y que se ven afectados por graves problemas de seguridad ${ }^{15}$. Y, en consecuencia, instaba a los Estados Miembros a tomar medidas penales y administrativas en la lucha contra este fenómeno $^{16}$.

Las medidas solicitadas por el CSNU pueden agruparse en varias categorías $^{17}$ : impedir la libre circulación de los terroristas mediante controles fronterizos eficaces y controlar la falsificación de documentos de identidad y de viaje, así como la utilización fraudulenta de dichos documentos; reforzar la coordinación entre los Estados en el intercambio de información sobre las actividades o los movimientos de combatientes terroristas extranjeros, especialmente con sus Estados de residencia o nacionalidad, por medio de mecanismos bilaterales o multilaterales; una mayor cooperación para prevenir la radicalización de potenciales combatientes, así como el apoyo para frenar la financiación y el reclutamiento de tales combatiente y, por último, la tipificación como delitos graves no sólo la financiación o el apoyo logístico y financiero a los grupos terroristas, sino también: a) el viaje o la tentativa de viaje a un tercer país con el propósito de colaborar en la comisión de actos terroristas o proporcionar o recibir adiestramiento; b) la financiación de tales viajes y; c) la organización o facilitación de dichos viajes.

Junto a la «desconcertante simbiosis» que se produce entre los términos «combatiente»y «terrorista» hasta ahora excluyentes ${ }^{18}$, la Resolución 2178 ha sido objeto de crítica por otras dos razones. La primera de ellas es que constituye una suerte de «resolución normativa instantánea» con la que se crean para los Estados obligaciones ex novo que no están basadas en una práctica previa ${ }^{19}$. La segunda es que, pese a reiterar la importancia del respeto de los derechos humanos, supone un adelantamiento de la acción punitiva en términos tan genéricos que deja la puerta abierta para que los Estados desarrollen legislaciones demasiado restrictivas o desproporcionadas $^{20}$.

${ }^{15}$ Consejo de Seguridad de Naciones Unidas, Resolución 2178, cit. supra.

16 GARrido MuÑOZ 2014: 303-307.

17 López-Jacoiste, cit. Supra: 49.

18 JiMÉNEZ GARCÍA 2016: 284.

19 JiMÉNEZ GARCÍA, cit. supra: 279.

${ }^{20}$ MARRERo Rocha 2016: 587; Informe del Relator Especial sobre la promoción y protección de los derechos humanos en la lucha contra el terrorismo, Doc. A/70/371, pp. 8-9. Disponible en: http://www.un.org/en/ga/search/view_doc.asp?symbol=A/70/371\&re ferer=http://www.un.org/es/documents/index.html\&Lang=S (Consultado el 24 de agosto de 2017). 


\section{El recurso a las medidas penales en el ámbito europeo}

El tercer informe sobre la aplicación de la Resolución $2178^{21}$ destaca la falta de tipificación de los delitos referentes a los combatientes terroristas extranjeros y la falta de cooperación entre los Estados de Asia Sudoriental (incluida Filipinas). Además, muestra su preocupación por nuevas leyes antiterroristas que buscan evitar los viajes al extranjero de presuntos terroristas mediante detenciones y arrestos domiciliarios sin acusación penal ni revisiones judiciales por periodos de hasta 2 años que pueden ser alargadas indefinidamente ${ }^{22}$.

En el ámbito europeo, por el contrario, encontramos un amplio desarrollo normativo en aplicación de la resolución onusiana. Apenas transcurridos dos meses desde la publicación de la Resolución 2178, el Comité de Expertos sobre Terrorismo del Consejo de Europa (CODEXTER) iniciaba el examen del fenómeno de los combatientes terroristas extranjeros. Como primera sugerencia al Comité de Ministros de esta organización, el CODEXTER planteó la creación de un comité ad hoc encargado de elaborar el borrador de un Protocolo Adicional al Convenio del Consejo de Europa para la prevención del terrorismo ${ }^{23}$. La idea fue acogida favorablemente y el 21 de enero de 2015 el Comité de Ministros creaba el Committee on Foreign Terrorist Fighters and Related Issues (COD-CTE) «with a view to the possible adoption of the draft Additional Protocol to the Council of Europe Convention on the Prevention of Terrorism $\rangle^{24}$.

Tras varios meses de negociaciones se adopta en Riga el Protocolo adicional del Convenio del Consejo de Europa para la prevención del terrorismo (en adelante, Protocolo de Riga) ${ }^{25}$. En este instrumento se criminalizan ciertas conductas asociadas a los combatientes terroristas extranjeros tales como la participación en una asociación o grupo con fines terroristas (art. 2), recibir entrenamiento para cometer actos de terrorismo (art. 3), viajar al extranjero con fines de terrorismo (art. 4), la financiación de dichos viajes (art. 5) y, por

21 Tercer Informe sobre la aplicación de la resolución 2178 (2014) del Consejo de Seguridad por los Estados afectados por los combatientes terroristas extranjeros, Doc. S/2015/975. Disponible en: http://www.un.org/en/sc/ctc/docs/2015/N1545990_ES.pdf (Consultado el 24 de agosto de 2017).

${ }^{22}$ Véase ibid., p. 38.

${ }_{23}$ Véase Convenio del Consejo de Europa para la prevención del terrorismo (ETS núm. 196), hecho en Varsovia el 16 de mayo de 2005.

${ }^{24}$ Council of Europe action against radicalisation leading to terrorism, Doc. SG/Inf (2015) 4, CM (2015) 19-rev, DD (2015) 95, de 21 de enero de 2015. Disponible en: https://search.coe.int/cm/Pages/result_details.aspx?ObjectID $=09000016805$ c4600 (Consultado el 24 de agosto de 2017).

25 Para un análisis del borrador del Protocolo véase PiACENTE 2015: 12-15. 
último, la organización o facilitar de cualquier otra forma estos viajes (art. 6). A ello se suman las previsiones de intercambio de información entre los Estados en la persecución de estos delitos (art. 7) y de respeto de los derechos humanos en la aplicación de este instrumento (art. 8).

En cuanto a la UE, el Consejo de Asuntos de Justicia e Interior apoyaba en junio de 2013 un paquete de 22 medidas sugeridas por el Coordinador de lucha contra el terrorismo de la UE para hacer frente al fenómenos de los combatientes terroristas extranjeros ${ }^{26}$. Este paquete de medidas fue la base de posteriores avances en el reforzamiento del Marco Schengen, en el famoso sistema PNR (Passenger Name Record), el intercambio de información, la cooperación operacional y, en el mismo camino que el Protocolo de Riga, en la armonización de tipos delictivos para criminalizar las conductas típicas de los combatientes terroristas extranjeros ${ }^{27}$.

Precisamente es este último aspecto, el de las medidas de justicia penal, el que llevó al Consejo Europeo a solicitar a la Comisión Europea una revisión de la entonces Decisión marco sobre lucha contra el terrorismo, en línea con las advertencias de Eurojust sobre lagunas en el enjuiciamiento de los combatientes terroristas extranjeros y la necesidad de un enfoque penal común en los Estados miembros ${ }^{28}$. Fruto de esta revisión es la Directiva relativa a la lucha contra el terrorismo aprobada el 15 de marzo de $2017^{29}$. Con la vista puesta en la Resolución 2178 y el Protocolo de Riga, esta Directiva «establece normas mínimas relativas a la definición de las infracciones penales y las sanciones en el ámbito de los delitos de terrorismo, los delitos relacionados con un grupo terrorista y los delitos relacionados con actividades terroristas, así como medidas de protección, apoyo y asistencia a las víctimas del terrorismo» (art. 1).

La nueva Directiva relativa a la lucha contra el terrorismo tiene su base legal en el art. 83.1 del Tratado de Funcionamiento de la Unión Europea $(\mathrm{TFUE})^{30}$ según el cual «el Parlamento Europeo y el Consejo podrán establecer, mediante directivas adoptadas con arreglo al procedimiento legislativo

${ }^{26}$ Consejo de la Unión Europea, reunión en Asuntos de Justicia e Interior, Press Release, 6 y 7 de junio de 2013, p. 17. Disponible en: http:/www.consilium.europa.eu/ueDocs/cms_Data/docs/pressData/en/jha/137407.pdf (Consultado el)

27 De KERChOVE y HÖHN 2016: 305-319.

28 Anti-terrorism: Eurojust in the European Parliament - Foreign Fighters in Focus, Press Release. Disponible en: http://www.eurojust.europa.eu/press/News/News/Pages/2014/2014-11-06_LIBE-Committee-debate.aspx (Consultado el 24 de agosto de 2017).

29 Directiva (UE) del Parlamento Europeo y del Consejo de 15 de marzo de 2017 relativa a la lucha contra el terrorismo y por la que se sustituye la Decisión marco 2002/475/ JAI del Consejo y se modifica la Decisión 2005/671/JAI del Consejo, DOUE L 88/6.

${ }^{30}$ Versión Consolidada del Tratado de Funcionamiento de la Unión Europea, de 26 de octubre de 2012, DOUE C 326/47. 
ordinario, normas mínimas relativas a la definición de las infracciones penales y de las sanciones en ámbitos delictivos que sean de especial gravedad y tengan una dimensión transfronteriza derivada del carácter o de las repercusiones de dichas infracciones o de una necesidad particular de combatirlas según criterios comunes. Estos ámbitos delictivos son los siguientes: el terrorismo [...]».

Las conductas a criminalizar por los Estados miembros son agrupadas en tres categorías: delitos de terrorismo (art. 3), delitos relacionados con un grupo terrorista (art. 4) y delitos relacionados con actividades terroristas (arts. 5 a 12). Es en esta última categoría donde aparecen las principales novedades de la Directiva: la recepción de adiestramiento para el terrorismo (art. 8), viajar con fines terroristas (art. 9), organizar o facilitar viajes con fines terroristas (art. 10) y la financiación de dichos actos (art. 11). A ello se suma una nueva previsión para la asistencia y apoyo a las víctimas del terrorismo (art. 24) en línea con la Directiva sobre los derechos, el apoyo y la protección de las víctimas de delitos ${ }^{31}$.

\section{El impacto de la criminalización de ciertas conductas en las libertades fundamentales}

Al comienzo de este apartado ya se mencionaba la crítica al «adelantamiento de la acción punitiva» que supone la Resolución 2178 y el efecto dañino que puede tener en los derechos humanos. Las obligaciones que impone a los Estados (y que pueden verse reflejadas en los instrumentos del Consejo de Europa y de la UE ya mencionados) pueden interferir gravemente en un amplio catálogo de derechos y libertades: la libertad de movimiento; el derecho a volver al país de tu nacionalidad; la libertad de entrada en un Estado conforme a las normas de asilo y refugio; la retirada de la nacionalidad; el derecho a la vida privada y la familia en tanto que estas medidas pueden afectar a individuos que se vean privados de entrar en el territorio de su residencia y en el cual habitan sus familias; el derecho a la privacidad por el almacenamiento y uso de información en las actividades de control de fronteras; la prohibición de discriminación en las actividades de «profiling»; la prohibición de detenciones arbitrarias; la violación del principio de nonrefoulement; los principios de legalidad, no retroactividad y doble incriminación del ordenamiento penal, así como los derivados del derecho a un juicio justo ${ }^{32}$.

${ }^{31}$ Directiva 2012/29/UE del Parlamento Europeo y del Consejo, de 25 de octubre de 2012, por la que se establecen normas mínimas sobre los derechos, el apoyo y la protección de las víctimas de delitos, y por la que se sustituye la Decisión marco 2001/220/JAI del Consejo, DOUE L 315/57.

32 CONTE 2016: 286. 
Para algún autor estos efectos se acentuarían a la hora implementar el «sloppy drafting» de la Resolución 2178 en los ordenamientos regionales ${ }^{33}$. Como no tarda en recordar, ciertas conductas que los Estados deben criminalizar forman parte de la rutina diaria de muchas personas con objetivos legítimos, tales como viajar al extranjero ${ }^{34}$. Algunas organizaciones no gubernamentales como Amnistía Internacional o la Comisión Internacional de Juristas comparten también el temor de que estas medidas, redactadas en términos demasiado generales y poco precisos, desemboquen a la hora de ser aplicadas por los Estados en una violación de la libertad de movimiento y del principio de legalidad penal «which requires that laws are clear and accessible and that their application in practice is sufficiently foreseeable, and which has been affirmed by the European Court of Human Rights as an essential element of the rule of law and an important protection against arbitrariness $\rangle^{35}$.

Los participantes en el proceso de creación de los dos instrumentos europeos ya comentados eran desde luego de que estas medidas podían contribuir a «la tendencia a objetivar la responsabilidad penal que ha dado lugar a que en ocasiones algunos Estados eludan la intencionalidad del acto y basen la sanción penal en el mero hecho de viajar a zonas calificadas como conflictivas o prohibidas a menos que se invoquen motivos legítimos, como la prestación de ayuda humanitaria, la realización de labores de periodismo o visitas de familiares $»^{36}$.

Tal y como como indica algún autor, no queda claro cómo distinguir entre quienes viajan al extranjero con fines legítimos de quienes lo hacen con un fin terrorista aún por manifestar sin recurrir a un «profiling» étnico ${ }^{37}$. La implementación de la Resolución 2178 puede así llevar a la prohibición de facto de viajar a ciertos países con áreas afectadas por actividades terroristas $^{38}$. Esta preocupación no es meramente teórica. Si acudimos a las últimas modificaciones de la ley antiterrorista australiana (y en concreto la conocida como «Foreign Fighter Bill»), cualquier persona que «enters, or remains, in an area of a foreign country» que sea declarada por el Ministerio de Asuntos Exteriores como una zona en la cual «a listed terrorist organisation is engaging in hostile activity» comete una ofensa criminal, a no

33 SCHEININ 2015.

${ }^{34}$ Véase ibid.

35 Amnistía Internacional, Submission of Amnesty International and the International Cpmmission of Jurists to the Council of Europe Committee of Experts on Terrorism (CODEXTER): Draft Additional Protocol to the Council of Europe Convention on the Prevention of Terrorism, AI Index: IOR 60/1393/2015, 7 de abril de 2015, p. 2.

36 JiMÉNEZ GARCÍA, cit. supra.: 290.

37 Ambos 2 de octubre de 2014.

38 Véase ibid. 
ser que la persona adopte esta conducta por razones legítimas, como las indicadas anteriormente ${ }^{39}$.

En el caso del Protocolo de Riga, a lo largo de su articulado se repite que el acto debe ser cometido «unlawfully and intentionally». Más concretamente, el Consejo de Europa reconocía las implicaciones para la libertad de movimiento y el principio de legalidad penal en el Informe explicativo de este protocolo ${ }^{40}$. Ya en sus primeras páginas, referidas al preámbulo, recuerda que «while there are posible restrictions to some of these rights provided by the aforesaid international human rights instruments, a number of rights, such as prohibition against the retrospective operation of criminal laws and freedom from torture and other cruel, inhuman or degrading treatment or punishment, are absolute and non-derogable» ${ }^{41}$.

Tras aclarar que la obligación de tipificar las conductas incluidas no implica que se creen crímenes específicos (basta con que sean incluidas como actos preparatorios o tentativas), el informe pasa a explicar en qué consiste la expresión «unlawfully and intentionally». En primer lugar, con respecto a la ilegalidad (unlawfulness) se refiere a las conductas realizadas sin ninguna autoridad o que no están cubiertas por previsiones legales u otros principios o intereses que justifiquen la exclusión de la responsabilidad criminal en el ordenamiento doméstico de que se trate ${ }^{42}$.

En segundo lugar, la ofensa criminal debe ser cometida «intencionalmente» (intentionally). Aquí se indica que el contenido exacto de la «intencionalidad» debe ser dejado, conforme a la práctica habitual del Consejo de Europa en la redacción de instrumentos de derecho penal, a la interpretación de los ordenamientos nacionales ${ }^{43}$. A lo cual se sumaría un elemento subjetivo adicional: el fin terrorista (conforme a los artículos 2 a 4) o el conocimiento del fin terrorista (conforme a los artículos 5 y 6 ). Una redacción que recuerda de algún modo al esquema general de los crímenes del derecho internacional penal que distingue, dentro del elemento subjetivo del crimen (mens rea) entre la intención criminal general en la perpetración del acto (dolus) y la intención específica del autor (dolus specialis) ${ }^{44}$. Y termina

${ }^{39}$ Counter-Terrorism Legislation Amendment (Foreign Fighter) Act 2014, Núm. 116. Disponible online en: https://www.legislation.gov.au/Details/C2014A00116/Download (Consultado el 24 de agosto de 2017).

${ }^{40}$ Council of Europe, Explanatory Report to the Additional Protocol to the Council of Europe Convention on te Prevention of Terrorism (ETS núm. 217), de 22 de octubre de 2015. Disponible en: https://rm.coe.int/168047c5ec (Consultado el 24 de agosto de 2017).

41 Véase ibid., p.3.

42 Véase ibid., p.4.

43 Véase ibid., p. 4.

44 Para un análisis más profundo de estos elementos en el caso del terrorismo véase WILMSHURST 2010: 347. 
recordando el informe que los Estados «shall take into account that Articles 2 to 6 criminalise behaviour at a stage preceding the actual commission of a terrorist offence but already having the potential to lead to the commission of such acts. The conditions under which the conduct in question is criminalised need to be foreseeable with legal certainty [...]. As always, the principle of the presumption of innocence should be respected, and the burden of proof lies with the State» ${ }^{45}$.

Los arts. 3 y 4 del Protocolo de Riga, relativos a la recepción de adiestramiento y al viaje con fines terroristas, merecen una especial atención por el impacto que tienen en las libertades civiles. El Consejo de Europa se muestra consciente de que el adiestramiento puede tener lugar bien en persona bien a través de medios electrónicos como Internet y los graves perjuicios que puede ocasionar un exceso en la criminalización en el derecho a la libertad de expresión $^{46}$. Es por ello que en el informe se señala que «the mere fact of visiting websites containing information or receiving communications, which could be used for training for terrorism, is not enough to commit the crime of receiving training for terrorism under the Protocol. The perpetrator must normally take an active part in the training. An example would be the participation of the perpetrator in interactive training sessions via the Internet».

El impacto que tiene la criminalización de los viajes al extranjero en el derecho a la libertad de movimiento es más que evidente. El Consejo de Europa tomó nota de la protección de este derecho por el art. 2 del Protocolo $\mathrm{n}^{\circ} 4$ al Convenio para la protección de los Derechos Humanos y de las Libertades Fundamentales ${ }^{47}$ y por el art. 12 del Pacto Internacional de Derechos Civiles y Políticos ${ }^{48}$. No obstante, «the seriousness of the threat posed by foreign terrorist fighters» justificaría una restricción de este derecho en aras de la protección de la seguridad ${ }^{49}$. Para asegurar la compatibilidad de estas restricciones con los derechos humanos y el imperio de la ley, el Consejo de Europa indica que deben cumplirse dos requisitos para criminalizar esta conducta $^{50}$. En primer lugar, el propósito real del viaje debe ser cometer o participar en actos terroristas, o recibir o proveer adiestramiento para el terrorismo, en un Estado distinto al de la nacionalidad o residencia. En segundo lugar, el

45 Explanatory report, cit. supra.: 4-5.

46 Véase ibid., p. 6.

${ }^{47}$ Véase Protocolo ${ }^{\circ} 4$ al Convenio para la protección de los Derechos Humanos y de las Libertades Fundamentales (ETS Núm. 046), hecho en Estrasburgo el 16 de septiembre de 1965.

48 Pacto Internacional de Derechos Civiles y Políticos, hecho en Nueva York el 23 de marzo de 1976, UNTS 999. Disponible en: https://treaties.un.org/Pages/showDetails.aspx ?objid=0800000280004bf5\&clang=_en (Consultado el 24 de agosto de 2017).

49 Explanatory report, cit. supra., p.7.

50 Explanatory report, cit. supra., p. 7. 
crimen debe ser cometido de forma «intencional e ilegal», en los términos expuestos anteriormente. Y enfatiza: «Article 4 does not contain an obligation for Parties to introduce a blanket ban on, or criminalisation of, all travels to certain destinations $\rangle^{51}$.

Estas consideraciones fueron también abordadas por la Comisión de Libertades Civiles, Justicia y Asuntos de Interior del Parlamento Europeo (Comisión LIBE). La mayor claridad posible en la intencionalidad de quienes realizan un viaje al extranjero con supuestos fines terroristas fue uno de los principales focos de atención. Se insistía así en incluir que «pueda demostrarse objetivamente que la finalidad prevista de dicho viaje era cometer o colaborar en la comisión de cualquiera de un acto terrorista [...] cuando se cometa dolosamente ${ }^{52}$. Como puede verse en la redacción final del art. 9, se hace un especial hincapié en que el hecho se produzca «intencionadamente» pero se omite la referencia a que «pueda demostrarse objetivamente» la finalidad terrorista. De hecho, para encontrar esta referencia a las circunstancias objetivas hay que dirigirse a los considerandos de la Directiva y, en particular, a los números 8 y 17.

Las injerencias en la libertad de expresión, y particularmente en relación con la eliminación y bloqueo de contenidos en línea, fue otro de los caballos de batalla de la Comisión LIBE. Ella misma estuvo bloqueada por las distintas posiciones de sus miembros en cuanto a la dureza de las medidas de vigilancia y censura, lo cual fue superado con la inclusión expresa del «control judicial» en la propuesta ${ }^{53}$. La versión definitiva de la Directiva incluye que estas medidas «deberán establecerse por procedimientos transparentes y ofrecer garantías adecuadas, sobre todo para garantizar que se limiten a lo necesario y proporcionado y que los usuarios estén informados de su justificación. Las garantías relativas a la eliminación o al bloqueo incluirán asimismo la posibilidad de recurso judicial $\aleph^{54}$.

El intento de incluir un artículo sobre los Principios fundamentales relativos a la libertad de expresión no tuvo éxito ${ }^{55}$, si bien se contempla un genérico art. 23 relativo al respeto de los derechos y libertades fundamentales. En este ámbito el texto más claro de la Directiva no se encuentra en su articulado

${ }^{51}$ Explanatory report, cit, supra., p. 7.

${ }_{52}$ Parlamento Europeo, Comisión de Libertades Civiles, Justicia y Asuntos de Interior, Informe sobre la propuesta de Directiva del Parlamento Europeo y del Consejo relativa a la lucha contra el terrorismo, y por la que se sustituye la Decisión Marco 2002/475/ JAI del Consejo sobre lucha contra el terrorismo (COM(2015)0625C80386/2015-2015/0281/COD)), de 12 de julio de 2016. p. 40.

53 LÓPEZ-JACOISTE, cit. supra.: 65.

${ }^{54}$ Directiva relativa a la lucha contra el terrorismo, cit. supra., art. 11.

${ }^{55}$ Informe de la Comisión LIBE, cit. supra., p. 56. 
sino en su Considerando 35, en el cual se menciona la necesidad de respetar el derecho a la libertad y la seguridad; la libertad de expresión y de información; la libertad de asociación y de pensamiento, conciencia y religión; la prohibición de discriminación; así como los principios de legalidad y proporcionalidad de los delitos y las penas.

Las críticas sobre el impacto que tienen estos dos instrumentos en los derechos y libertades fundamentales parecen perder fuerza una vez que se examina la totalidad de estos textos, las garantías que en ellos se establecen y las explicaciones que los acompañan. Sin embargo, los riesgos no desaparecen. Una parte nada desdeñable sobre la forma y el sentido de la criminalización de estas conductas se encuentra en los considerandos de la Directiva y el informe explicativo del Protocolo. Las primeras líneas del informe nos recuerdan que «the text of this Explanatory Report does not constitute an instrument providing an authoritative interpretation of the Additional Protocol, although it may be of such nature as to facilitate the application of the provisions contained therein». El respeto a las libertades y derechos fundamentales dependerá así de cómo se implementen estas normas a nivel doméstico, y la supervisión política y judicial posterior que contribuya a encontrar el balance entre libertad y seguridad ${ }^{56}$. Y, desde luego, no falta quienes se muestran escépticos sobre el respeto de estos principios y derechos a la hora de implementar a nivel nacional un instrumento que, en sí mismo, no ofrece suficiente claridad al respecto ${ }^{57}$.

\section{CONCLUSIONES}

La amenaza de los combatientes terroristas extranjeros se ha convertido en una dolorosa realidad para Europa, al igual que para Filipinas. La respuesta normativa a nivel internacional contra este fenómeno ha sido liderada por el CSNU con la Resolución 2178, aunque hasta la fecha Filipinas no ha tomado medidas expresas para su implementación. Sí lo han hecho, a nivel regional europeo, el Consejo de Europa y la UE. Entre las principales novedades cabe destacar un refuerzo en la armonización de los tipos penales para incluir ciertas conductas típicas en las que incurren los combatientes terroristas extranjeros tales como los viajes al extranjero con fines terroristas o la recepción de adiestramiento en técnicas terroristas, ya sea en persona o a través de Internet. Esta respuesta normativa ha sido duramente criticada por ser un adelantamiento de la acción punitiva que permitiría a los Estados, a la hora de implementar estos instrumentos, restringir numerosos derechos humanos y libertades fundamentales, y en especial la libertad de movimiento

\footnotetext{
56 GARrido MuÑoz 2016. 351.

57 SCHEININ, cit. supra.; BoBEK 2014: 268.
} 
y la libertad de expresión. En este aspecto la labor de los tribunales nacionales en el control del balance entre libertad y seguridad, y en última instancia del Tribunal Europeo de Derechos Humanos y del Tribunal de Justicia de la Unión Europea, se revela más importante que nunca. En el caso de Filipinas estas medidas pueden resultar útiles en la lucha contra un fenómeno que está azotando duramente su sociedad, pero debe tener en cuenta las restricciones que comporta y desarrollar las garantías adecuadas que permitan cumplir con los compromisos de protección de los derechos humanos y las libertades fundamentales.

\section{BIBLIOGRAFÍA}

Ambos, K., «Our Terrorists, Your Terrorists? The United Nations Security Council Urges States to Combat Foreign Terrorist Fighters, But Does Not Define Terrorism», EJIL Talk, (2 de octubre de 2014). Disponible en: https://www.ejiltalk. org/our-terrorists-your-terrorists-the-united-nations-security-council-urges-states-to-combat-foreign-terrorist-fighters-but-does-not-define-terrorism/ (Consultado el 24 de agosto de 2017).

Boвeк, M., «The Fight against Terror and the Space of Individual Freedom: A (Classic) Word of Caution», en Govaere, I. y Poli, S., EU Management of Global Emergencies: Legal Framework for Combating Threats and Crises, Leiden, (Brill Nijhoff, 2014): 268.

Charithie, J., «Daesh and the Philippines: Distant but virulent Insporation» en Asian Conflicts Report: The Geopolitics of Extremisms: ISIS and Asia, Geneva Center for Security Policy, (Agosto de 2015): 6-7. Disponible en: http://www.gcsp.ch/ download/4450/104352 (Consultado el 24 de agosto de 2017).

Conte, A., «States' Prevention and Responses to the Phenomenon of Foreign Fighters against the Backdrop of International Human Rights Obligations», en De Guttry, A., Capone, F. y Paulussen, C., Foreign Fighters under International Law and Beyond, Springer, T.M.C Asser Press, La Haya, 2016, p. 286.

De Kerchove, G. y HöHn, C., «The Regional Answers and Governance Structure for Dealing with Foreign Fighters: The Case of the EU», en De Guttry, A., Capone, F. y Paulussen, C., Foreign Fighters under International Law and Beyond, Springer, T.M.C Asser Press, La Haya, 2016, pp. 305-319.

ESMAQUEL, P., «Philippines offers help in US war against ISIS», Rappler, 25 de septiembre de 2014. Disponible en http://www.rappler.com/nation/70162-philippines-help-united-states-isis (Consulado el 24 de agosto de 2017).

Garrido MuÑoz, A., «Additional Protocol to the Council of Europe Convention of the Prevention of Terrorism», European Papers, Highlight, Vol. 1, Núm. 1, 2016, p. 351.

— «Un nuevo cortocircuito legislativo en Naciones Unidas: la Resolución 2178 (2014) del Consejo de Seguridad de Naciones Unidas relativa a los combatientes terroristas extranjeros», Revista Española de Derecho Internacional, Vol. 67, Núm. 2, 2014, pp. 303-307. 
Gerstein, J. y Scholtes, J., «Comey Warns of Post-ISIL Terrorist Diaspora», Politico, 27 de septiembre de 2016. Disponible en: http://www.politico.com/ story/2016/09/islamic-state-diaspora-james-comey-228782 (Consultado el 24 de agosto de 2017).

GunARATnA, R., «Marawi: a Game Changer in Terrorism in Asia», Counter Terrorist Trends and Analyses: A Journal of the International Centre for Political Violence and Terrorism Research, Vol. 9, Núm. 7, 2017, p. 4.

Koruth SAmuel, T., «Radicalisation in Southeast Asia: A Selected Case Study of Daesh in Indonesia, Malaysia and The Philippines», The Southeast Asia Regional Centre for Counter-Terrorism, 2016, pp. 81-100. Disponible en: https:/www. unodc.org/documents/southeastasiaandpacific/Publications/2016/Radicalisation_SEA_2016.pdf (Consultado el 24 de agosto de 2017)

LÓPEZ-JACOISTE, E., «La Unión Europea ante los Combatientes Terroristas Extranjeros», Revista de Estudios Europeos, Núm. 67, 2016, p. 51.

MArrero Rocha, I., «Los Combatientes Terroristas Extranjeros de la Unión Europea a la Luz de la Resolución 2178 (2014) del Consejo de Seguridad de las Naciones Unidas, Revista de Derecho Comunitario Europeo, Núm. 54, (2016): 587; Informe del Relator Especial sobre la promoción y protección de los derechos humanos en la lucha contra el terrorismo, Doc. A/70/371: 8-9. Disponible en: http://www.un. org/en/ga/search/view_doc.asp?symbol=A/70/371\&referer=http://www.un.org/es/ documents/index.html\&Lang=S (Consultado el 24 de agosto de 2017).

Paulussen, C., «Repressing the Foreign Fighters Phenomenon and Terrorism in Western Europe: Towards an Effective Response Based on Human Rights», ICCT Research Paper, (2016): 3. Disponible en: https://icct.nl/wp-content/ uploads/2016/11/ICCT-Paulussen-Rule-of-Law-Nov2016-3.pdf (Consultado el 24 de agosto de 2017).

Piacente. N., «The Contribution of the Council of Europe to the Fight Against Foreign Terrorist Fighters: The Draft Additional Protocol to the Council of Europe Convention on the Prevention of Terrorism 2005», The European Criminal Law Associations' Forum, Núm. 1, (2015): 12-15.

RESSA, M.A., «Senior Abu Sayyaf leader swears oath to ISIS», Rappler, (4 de agosto de 2014). Disponible en: http://www.rappler.com/nation/65199-abu-sayyaf-leader-oath-isis (Consultado el 24 de agosto de 2017).

ScheInIN, M., «The Council of Europe's Draft Protocol on Foreign Fighters is Fundamentally Flawed», (2015). Disponible en: https://www.justsecurity.org/21207/ council-europe-draft-protocol-foreign-terrorist-fighters-fundamentally-flawed/ (Consultado el 24 de agosto de 2017).

Singh, B. y RAmakrishnA, K., «Islamic State's Wilayah Philippines: Implications for Southeast Asia», RSIS Commentary, Núm. 187, (2016).

Wilmshurst, E., «Transnational Crimes, Terrorism and Torture», en Cryer, R., Friman, H., Robinson, D. Y Wilmshurst, E. (eds.), An Introduction to International Criminal Law and Procedure, Cambridge University Press, (2010): 347.

ANTI-TERRORISM: Eurojust in the European Parliament - Foreign Fighters in Focus, Press Release. Disponible en: http://www.eurojust.europa.eu/press/News/News/ 
Pages/2014/2014-11-06_LIBE-Committee-debate.aspx (Consultado el 24 de agosto de 2017).

Consejo de Seguridad de Naciones Unidas, Resolución 2178 de 24 de septiembre de 2014, Doc. S/RES/2178. Disponible en: https://documents-dds-ny.un.org/doc/ UNDOC/GEN/N14/548/02/pdf/N1454802.pdf?OpenElement (Consultado el 24 de agosto de 2017).

CONVENIO DEl CONSEJO de EUROPA PARA LA PREVENCIÓN DEL TERRORISMO (ETS núm. 196), hecho en Varsovia el 16 de mayo de 2005.

COUNCIL OF EUROPE ACTION AGAINST RADICALISATION LEADING TO TERRORISM, Doc. SG/Inf (2015) 4, CM (2015) 19-rev, DD (2015) 95, de 21 de enero de 2015. Disponible en: <https://search.coe.int/cm/Pages/result_details.aspx?ObjectID= 09000016805c4600> (Consultado el 24 de agosto de 2017).

Council of Europe, Explanatory Report to the Additional Protocol to the Council of Europe Convention on te Prevention of Terrorism (ETS núm. 217), de 22 de octubre de 2015. Disponible en: https://rm.coe.int/168047c5ec (Consultado el 24 de agosto de 2017).

Counter-Terrorism Legislation Amendment (Foreign Fighter) Act 2014, Núm. 116. Disponible online en: https://www.legislation.gov.au/Details/C2014A00116/ Download (Consultado el 24 de agosto de 2017).

INTERPOL, «Los combatientes extranjeros, eje central de una reunión de INTERPOL sobre la lucha contra el terrorismo». Disponible en: https://www.interpol.int/es/ Centro-de-prensa/Noticias/2013/N20130712/ (Consultado el 24 de agosto de 2017).

Munich Security Conference, «Munich Security Report 2016: Boundless Crises, Reckless Spoilers, Helpless Guardians», p. 21. Disponible en: https:/www.securityconference.de/fileadmin/MunichSecurityReport/MunichSecurityReport 2016.pdf (Consultado el 24 de agosto de 2017).

Pacto Internacional de Derechos Civiles y Políticos, hecho en Nueva York el 23 de marzo de 1976, UNTS 999. Disponible en: https://treaties.un.org/Pages/ showDetails.aspx?objid=0800000280004bf5\&clang=_en (Consultado el 24 de agosto de 2017).

Protocolo $\mathrm{N}^{\circ} 4$ al Convenio para la PRotección de los Derechos Humanos y de Las Libertades Fundamentales (ETS Núm. 046), hecho en Estrasburgo el 16 de septiembre de 1965 .

TERCER INFORME SOBRE LA APLICACIÓN DE LA RESOLUCiÓN 2178 (2014) del Consejo de Seguridad por los Estados afectados por los combatientes terroristas extranjeros, Doc. S/2015/975. Disponible en: http://www.un.org/en/sc/ctc/docs/2015/ N1545990_ES.pdf (Consultado el 24 de agosto de 2017). 